\title{
Wavelength-tunable mode-locked Yb-doped fiber laser based on nonlinear Kerr beam clean-up effect
}

\author{
Shanchao Ma (马善超 $)^{1}$, Baofu Zhang (张宝夫 $)^{2^{*}}$ ， Qiurun He (何秋润) ${ }^{1}$, Jing Guo (郭 靖) $)^{3}$, and Zhongxing Jiao (焦中兴) ${ }^{1^{* *}}$ \\ ${ }^{1}$ School of Physics, Sun Yat-sen University, Guangzhou 510275, China \\ ${ }^{2}$ School of Materials Science and Engineering, Dongguan University of Technology, Dongguan 523808, China \\ ${ }^{3}$ School of Opto-Electronics, Beijing Institute of Technology, Beijing 100081, China
}

*Corresponding author: zhangbf5@mail.sysu.edu.cn

${ }^{* *}$ Corresponding author: jiaozhx@mail.sysu.edu.cn

Received November 25, 2021 | Accepted January 19, २०22 | Posted Online February 11, २०22

\begin{abstract}
We demonstrate a novel approach to achieve wavelength-tunable ultrashort pulses from an all-fiber mode-locked laser with a saturable absorber based on the nonlinear Kerr beam clean-up effect. This saturable absorber was formed by a single-mode fiber spliced to a graded-index multimode fiber, and its tunable band-pass filter effect is described by a numerical model. By adjusting the bending condition of the graded-index multimode fiber, the laser could produce dissipative soliton pulses with their central wavelength tunable from $1040 \mathrm{~nm}$ to $1063 \mathrm{~nm}$. The pulse duration of the output laser could be compressed externally to $791 \mathrm{fs}$, and the signal to noise ratio of its radio frequency spectrum was measured to be $75.5 \mathrm{~dB}$.
\end{abstract}

Keywords: nonlinear Kerr beam clean-up effect; tunable wavelength; mode-locking; numerical simulation. DOI: 10.3788/COL202220.041403

\section{Introduction}

Wavelength-tunable lasers are widely used in optical communication, detection, and remote sensing. Compared to tunable continuous-wave lasers, tunable mode-locked lasers can generate ultrashort pulses with high peak power and wide bandwidth. They can enable many important advances, such as highsensitivity optical absorption measurement, multi-photon microscopy, and super-continuum light source used in dense wavelength division multiplexing. Moreover, due to the high conversion efficiency, low temperature sensitivity, and compact structure of fiber lasers, tunable mode-locked fiber lasers ${ }^{[1-4]}$ have attracted extensive research interest.

Up to date, there are several typical approaches to build up wavelength-tunable mode-locked fiber lasers. The most common method is to add a tunable spectral filter into the laser cavity ${ }^{[1-4]}$. Among them, although chirped fiber Bragg gratings have been used to achieve wavelength tuning ${ }^{[1]}$, the tuning range is limited to a few nanometers. A Fourier domain programmable optical processor can also be used as a spectral filter ${ }^{[2]}$, but it was based on a two-dimensional liquid-crystal-on-silicon array, which cannot be easy to construct. Multimode interference (MMI)-based band-pass filters can also be used to achieve tunable output ${ }^{[3,4]}$, but the mode-locking operation based on nonlinear MMI is very sensitive to the length of graded-index multimode fiber (GIMF). Therefore, in order to meet the requirements of emerging applications, more attention should be paid to find out a simple and reliable solution for wavelength-tunable mode-locked fiber lasers.

The nonlinear Kerr beam clean-up (NL-KBC) effect occurs when a high-power laser propagates in the GIMF. With the increasing laser power or longer fiber length, energy of highorder modes (HOMs) can be irreversibly coupled into the fundamental mode (FM), where the nonlinear Kerr effect is the driving mechanism ${ }^{[5,6]}$. Based on this NL-KBC effect, we have constructed all-fiber saturable absorbers (SAs) with the structure of a long GIMF spliced to a short single-mode fiber (SMF) ${ }^{[7,8]}$. We experimentally investigated characteristics of these SAs ${ }^{[7]}$ and then demonstrated a mode-locked Yb-doped fiber laser using one of them ${ }^{[8]}$. We found that the bending of GIMF in this laser might lead to different transverse mode distributions of the beam with different wavelengths, resulting in a band-pass filter characteristic along with the NL-KBC effect. This may be a new approach to achieve wavelength-tunable output in the modelocked fiber lasers.

In this paper, an all-fiber wavelength-tunable mode-locked laser based on the NL-KBC effect is demonstrated. The tuning mechanism is theoretically studied by our numerical model. For this laser, mode-locking operation with a wide tunable range from $1040 \mathrm{~nm}$ to $1063 \mathrm{~nm}$ can be obtained. The output pulse duration can be compressed externally to $791 \mathrm{fs}$. 


\section{Theoretical Model and Numerical Simulation}

In this section, we will investigate the band-pass filter effect and saturable absorption property of an SA based on NL-KBC effect by a numerical model. In this model, the SA can be divided into four parts, which are centrally aligned (shown in Fig. 1): an input SMF, a short GIMF segment with a small radius of curvature, a long and straight GIMF segment, and an output SMF. A commercial GIMF (Nufern, GR-50/125-23HTA) is used here; both input and output SMFs have the same type (Nufern, Hi1060$\mathrm{XP})$. It is assumed that only the multimode-coupling effect introduced by the bending condition is considered in the short GIMF segment, while dispersion and nonlinear effects are considered in the long GIMF segment. The bending condition here refers to the curved length and curvature radius. Here, the mode field diameter of this SMF is close to the FM diameter of the GIMF. Therefore, we can obtain approximate solutions by assuming that all laser energy from the input SMF will be transferred to the excited FM energy in the GIMF, while only the FM energy at the end of the GIMF can be coupled into the output SMF. With this approximation, the transmittance of this SA can be defined as the ratio of FM energy at the end of the GIMF to the launched laser energy from the input SMF. The main theoretical method and some important assumptions are listed at the flowchart in Fig. 1(b).

In the short GIMF segment, we mainly focus the influences of bending condition on the transverse mode distribution of the laser beam with different wavelengths. For qualitative analysis, the bending of the GIMF can greatly affect mode propagation constants and, hence, the coupling coefficients between laser transverse modes ${ }^{[9]}$. Besides, mode propagation constants also depend on the laser wavelength. The bending condition and

(a)

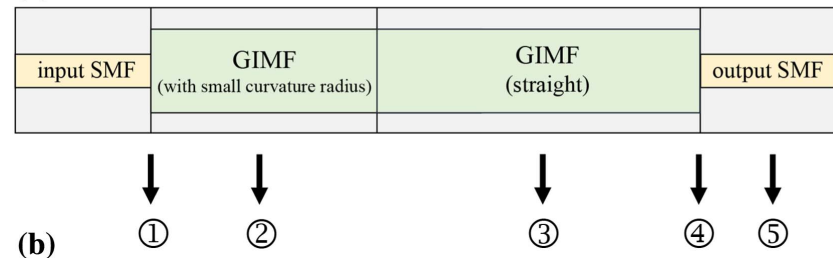

- All laser energy from the input SMF is transferred to the excited FM energy in the GIMF.

- Difference method is used to solve mode coupling equations along propagation distance with the constant radius bending, the energy proportion of FM at the end of short GIMF segment can be obtained.

- Massively parallel algorithm is used to solve the generalized multimode nonlinear Schrödinger equation, the energy proportion of FM at the end of long GIMF segment can be obtained.

- Only the FM energy at the end of GIMF can be coupled into the output SMF.

The transmittance, modulation depth and filter bandwidth of the saturable absorber can be defined and obtained from numerical results.

Fig. 1. (a) Structure of an SA based on the nonlinear Kerr beam clean-up effect and (b) flowchart of our numerical model. laser wavelength will have great influences on the output transverse mode distribution. In order to figure out these influences, numerical simulations are performed by solving the wave-coupling equation through a curved GIMF; this numerical method is similar to Sohn's work ${ }^{[10]}$. First, the transverse eigenmodes and their propagation constants of the GIMF can be obtained by analytically solving the waveguide eigen equation. Then, local transverse mode fields are matched at a succession of infinitesimal corner bends to calculate mode-coupling induced by the bend with a constant curvature radius. Finally, the energy proportion of different modes versus laser wavelength, propagation distance, and curvature radius can be obtained at the end of the GIMF. The detail of the three steps above can be found in pages 10-11, 35-46 of Ref. [10]. In this simulation, the length of short GIMF segment is set to be $40 \mathrm{~mm}$ with its curved length of 10 $40 \mathrm{~mm}$ and a curvature radius of $2-10 \mathrm{~mm}$. Moreover, it is assumed that the propagating laser beam is monochromatic, and only linearly-polarized (LP) modes are considered. Since the energy proportion of the LP01 mode at the end of the short GIMF segment has great influence on the transmittance of our SA (detailed in the following paragraph), this energy proportion with different bending conditions is shown in Fig. 2. For specific conditions, the energy proportion of the LP01 mode varies with the laser wavelength. These numerical results are in good agreement with the qualitative analysis mentioned above. As a result, the transverse mode distribution versus wavelength at the end of the short GIMF segment will serve as the input condition for numerical simulations in the long GIMF segment.

In the long GIMF segment, we mainly focus on the influences of input condition on the output laser characteristics. Since the main issue here is multimode nonlinear optical pulse propagation, numerical simulations are performed by solving a generalized multimode nonlinear Schrödinger equation using the massively parallel algorithm (MPA). The MPA numerical solver
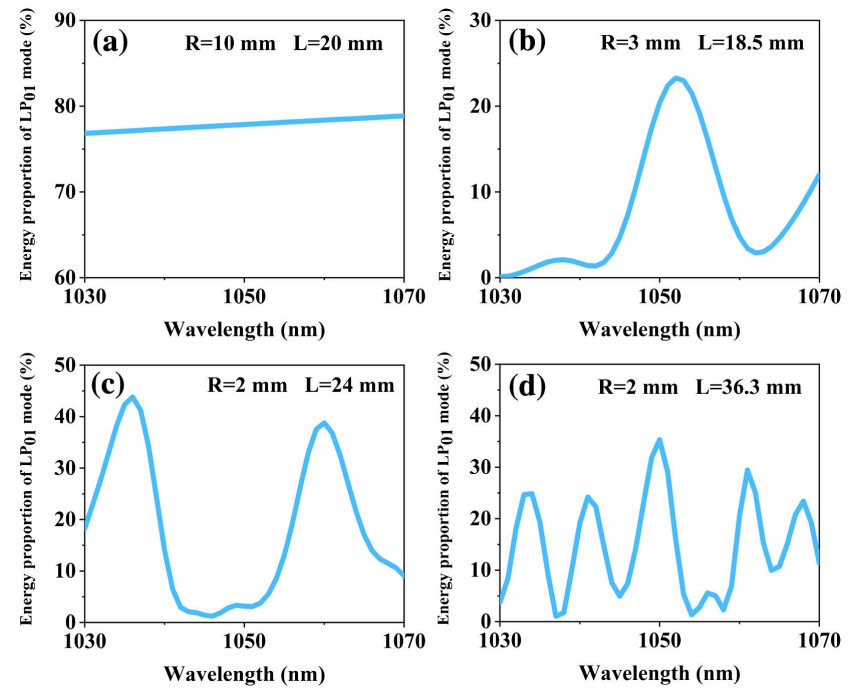

Fig. 2. Simulated energy proportion of the LP01 mode at the end of the short graded-index multimode fiber segment with different bending conditions. $R_{\text {, }}$ radius of curvature; $L$, curved length. 
we used is the same as that reported by Wright et al. ${ }^{[11]}$, and this solver edited in MATLAB is available online ${ }^{[12]}$. In our numerical model, only the first $10 \mathrm{LP}$ modes are considered. Moreover, the effects of self-phase modulation, stimulated Raman scattering (SRS), and the first five-order dispersions are included in the simulations, but the random linear distortions such as random radius perturbation and random index perturbation introduced by the imperfections or bending of GIMF are not included. The length of the long GIMF segment is set to be $2 \mathrm{~m}$, and the input laser pulse has a duration of $50 \mathrm{fs}$ with different pulse energy and central wavelength. For the situation of the short GIMF segment with $18.5 \mathrm{~mm}$ curved length and $3 \mathrm{~mm}$ curvature radius, as shown in Fig. 2(b), the energy proportion of the LP01 mode versus wavelength is simple and clear with a single peak at $1052 \mathrm{~nm}$. Therefore, in the following simulations, this situation is chosen as the input condition of the long GIMF segment. Firstly, we simulated and analyzed high-energy multimode laser pulse propagation through the long GIMF segment. When the laser pulse energy is set to be $50 \mathrm{~nJ}$, and its wavelength is $1052 \mathrm{~nm}$, the evolution of the laser transverse mode distribution along the GIMF is shown in Fig. 3(a). During the propagation, the proportion of the LP01 mode first increases from $23 \%$ to $52 \%$ and then gradually remains stable at $40 \%$; most of the HOMs have a lower proportion at the end of the GIMF. Therefore, part of the HOM energy is irreversibly coupled into the FM, which indicates that the NL-KBC effect occurs in this case. Then, we focus on the optical properties of this SA, which depend on the output laser characteristics after it propagates through this long GIMF segment. The relationship between transmittance of the SA and laser wavelength is presented in Fig. 3(b); the cases of input laser beam with high pulse energy $(50 \mathrm{~nJ})$ and low pulse energy $(0.001 \mathrm{~nJ})$ are both considered. As shown in Fig. 3(b), the transmittance of the SA versus wavelength has a similar shape to the energy proportion of the LP01 mode, as shown in Fig. 2(b), which results in a band-pass filtering effect. As shown in Fig. 2, this band-pass filter can be tunable since its central wavelength and bandwidth can be adjusted by changing the bending condition of the short GIMF segment and, hence, the input transverse mode distribution of the long GIMF segment. In addition, the transmittance with higher input pulse energy (red line) is higher than that with lower energy (blue line) at each wavelength, which confirms the saturable absorption effect of this SA. Finally, in order to further evaluate optical properties of the SA, its transmittance under different input pulse intensity is studied. The numerical results are presented in Fig. 3(c); the laser wavelength is chosen to be $1052 \mathrm{~nm}$, which is the central wavelength of the band-pass filter effect. The transmittance first grows monotonically as the input pulse energy increases and then decreases when the pulse intensity is high. The decrease of transmittance may be caused by SRS, which can lead to serious dissipation of the propagating laser power ${ }^{[6]}$. This assumption is also supported by the monotonically increasing transmittance when SRS is not included in the simulation. In order to evaluate the modulation depth and saturation intensity of this SA, the transmittance curve before the decrease can be fitted by
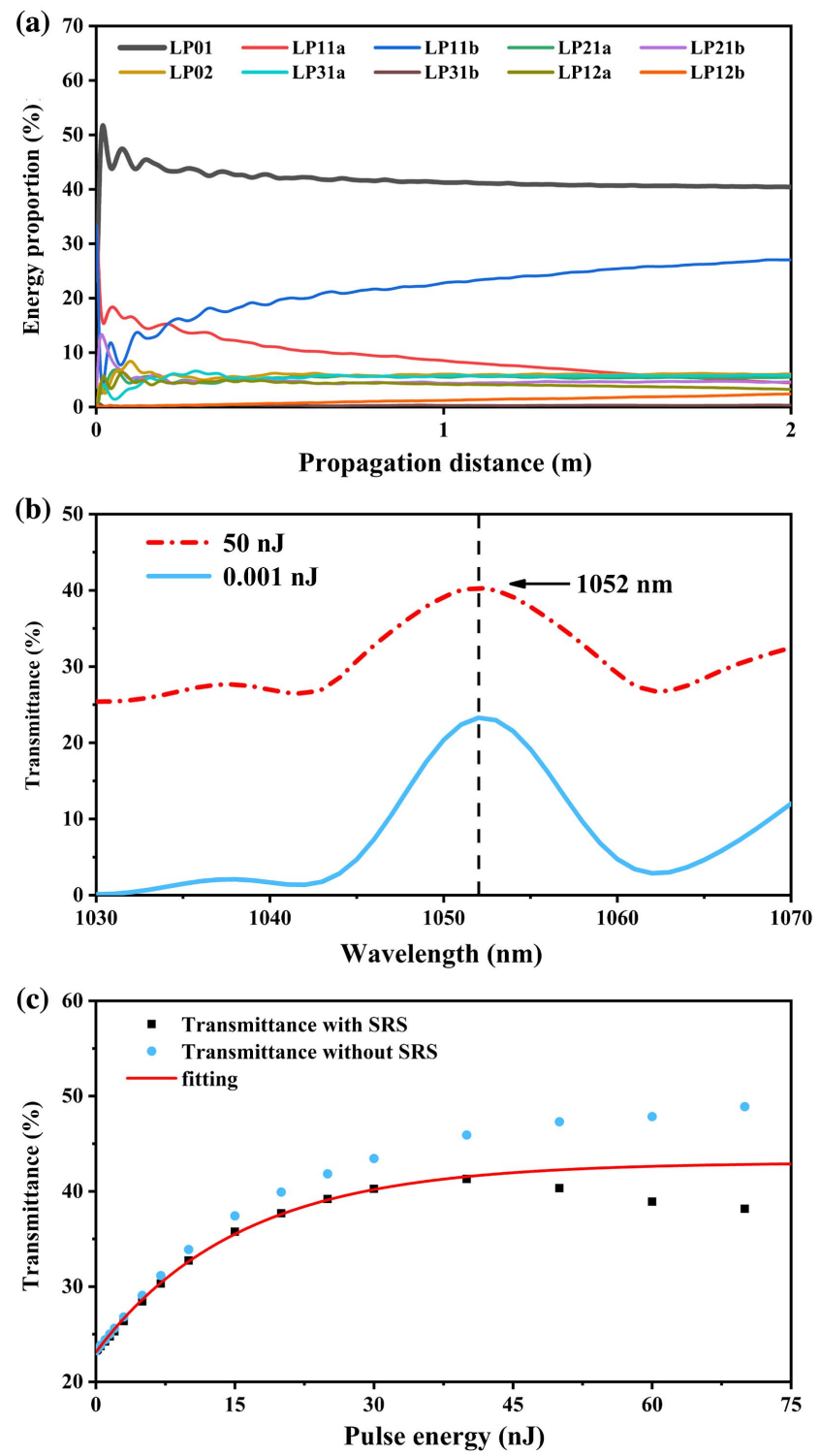

Fig. 3. Numerical results for the SA: (a) the evolution of modal energy proportion along the long GIMF segment when the laser wavelength is $1052 \mathrm{~nm}$; (b) the transmittance of the SA versus input laser wavelength when the pulse energy is $50 \mathrm{~nJ}$ (red line) and $0.001 \mathrm{~nJ}$ (blue line); (c) the transmittance of the SA versus input laser intensity when the laser wavelength is $1052 \mathrm{~nm}$.

$$
T=T_{0}+q \cdot\left[1-\exp \left(-\frac{I}{I_{\mathrm{SAT}}}\right)\right]
$$

where $T$ is the transmittance, $T_{0}$ is the initial transmittance, $q$ can be defined as the modulation depth, and $I$ and $I_{\mathrm{SAT}}$ are the input and saturation intensity, respectively. As shown in Fig. 3(c), the curve fits well with the numerical results; the initial transmittance, modulation depth, and saturation intensity are fitted to be $23.12 \%, 19.91 \%$, and $15.41 \mathrm{~nJ}$, respectively. These results further ensure the saturable absorption effect of the SA.

In conclusion of this section, a numerical model is used to investigate the optical properties of the SA based on the 
NL-KBC effect. The simulation results indicate that the bending condition plays an important role in the transverse mode distribution of the GIMF and hence the transmittance of the SA. The saturable absorption and band-pass filter effect with a tunable central wavelength of the SA can be achieved with specific bending conditions of the GIMF when the NL-KBC effect occurs. Therefore, this SA may be promising for constructing tunable mode-locked lasers.

\section{Experiments and Results}

In order to verify the tunable band-pass filtering effect of the SA based on NL-KBC effect, we built up a mode-locked Yb-doped fiber laser with this SA. As shown in Fig. 4, the configuration of the laser is similar to that demonstrated in our previous work ${ }^{[8]}$, except that the polarization independent isolator (PI-ISO) used in the cavity had a wide bandwidth $(>50 \mathrm{~nm})$. In this laser, a $976 \mathrm{~nm}$ laser diode was used as the pump source, and its pump power was coupled into an Yb-doped fiber (Yb1200-4/125, LIEKKI) through a commercial wavelength division multiplexer. The length of this gain fiber was chosen to be $0.3 \mathrm{~m}$ in order to reduce the mode-locking threshold and increase the output power. After the $\mathrm{Yb}$-doped fiber, $10 \%$ of the mode-locked laser power was extracted from a coupler. The output port of the coupler was connected with an isolator to prevent the influence of reflected light. The function of the PI-ISO was to ensure the unidirectional operation of the laser and eliminate the possibility of nonlinear-polarization-evolution mode locking. Two polarization controllers (PCs) were used to optimize intracavity birefringence in order to achieve a stable mode-locking state. The SA was the same as what we mentioned in the simulation section. The length of GIMF is designed to be $2 \mathrm{~m}$, and it was placed between the two PCs. The total loss of the SA was measured to be $3.01 \mathrm{~dB}$. The bending condition of the short GIMF segment can be adjusted by two $X Y$-axis translation stages with resolution of $10 \mu \mathrm{m}$. All single-mode passive fibers used in this laser were the same type (HI-1060, Corning); the total cavity length was measured to be about $7.4 \mathrm{~m}$.

When the short GIMF segment was adjusted to a specific bending condition, the self-starting mode-locking operation

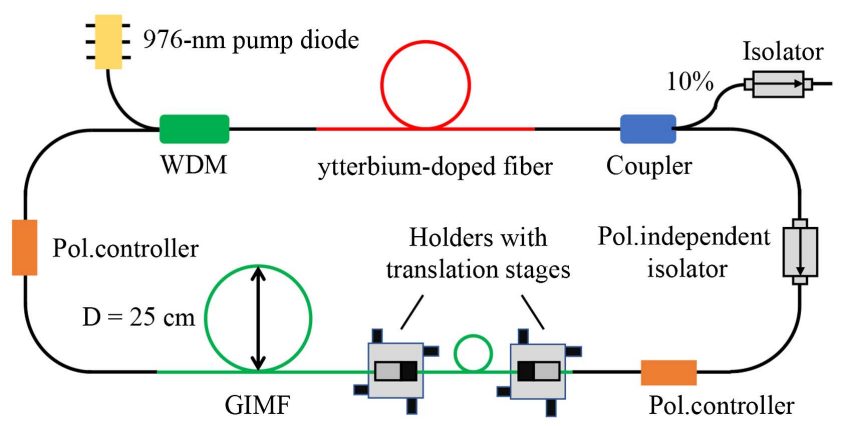

Fig. 4. Experimental setup of the tunable mode-locked Yb-doped fiber laser based on NL-KBC effect. WDM, wavelength division multiplexer; GIMF, gradedindex multimode fiber; Pol., polarization; $D$, diameter. could be achieved by only increasing the pump power. When the operating central wavelength was tuned to $1040 \mathrm{~nm}$, the single-pulse mode-locking state was obtained at the threshold of $60 \mathrm{~mW}$. With the pump power increasing to $160 \mathrm{~mW}$, the mode-locking state could be maintained, and the output pulse characteristics were measured. The evolution of laser output power and optical-to-optical conversion efficiency is shown in Fig. 5. The conversion efficiency is around $6 \%$ when the laser operates in the mode-locking state. With the pump power of $160 \mathrm{~mW}$, the average output power was measured to be $9.76 \mathrm{~mW}$, corresponding to the pulse energy of $0.35 \mathrm{~nJ}$. The output pulse trace and radio-frequency (RF) spectrum are presented in Fig. 6. The period of laser pulses was about $36 \mathrm{~ns}$, and the first peak of the RF spectrum was centered at $27.566 \mathrm{MHz}$, corresponding to the cavity length of $7.4 \mathrm{~m}$. The signal-to-noise ratio of this RF spectrum was measured to be $75.5 \mathrm{~dB}$; no multi-pulse or harmonic mode-locked signals were found in the RF spectrum. These results further confirmed that the laser was operating at a single-pulse mode-locking state. Figure 6(c) shows the laser output spectrum at the pump power of $160 \mathrm{~mW}$; its central wavelength was $1040 \mathrm{~nm}$ with a $20 \mathrm{~dB}$ bandwidth of $6.2 \mathrm{~nm}$. The spectrum presented a cat-ear-like trace, which is a typical characteristic of dissipative solitons from all-normal-dispersion fiber lasers. The autocorrelation trace of the output pulse was also measured [see Fig. 6(d)]; assuming a Gaussian pulse shape, the pulse duration was calculated to $25.7 \mathrm{ps}$. It could be compressed externally to $791 \mathrm{fs}$ using a single 1200 lines/mm grating.

In addition, wavelength-tunable mode-locking operation was realized in this laser. By adjusting the translation stages, different bending conditions of the GIMF can be achieved. As a result, the central wavelength of the output laser could be tuned from $1040 \mathrm{~nm}$ to $1063 \mathrm{~nm}$, while the mode-locking state could be maintained. The output spectra with different central wavelengths are presented in Fig. 7. They all showed a cat-ear-like trace, but their mode-locking thresholds are different, ranging from $60 \mathrm{~mW}$ to $330 \mathrm{~mW}$. Their spectral bandwidths are also different due to the various bending conditions of the GIMF, which

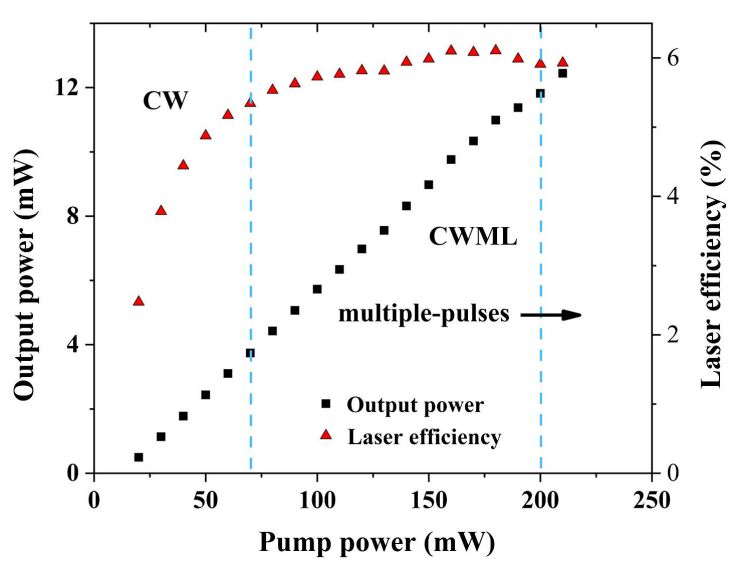

Fig. 5. Output power and conversion efficiency versus pump power. CW, continuous wave; CWML, CW mode-locking. 

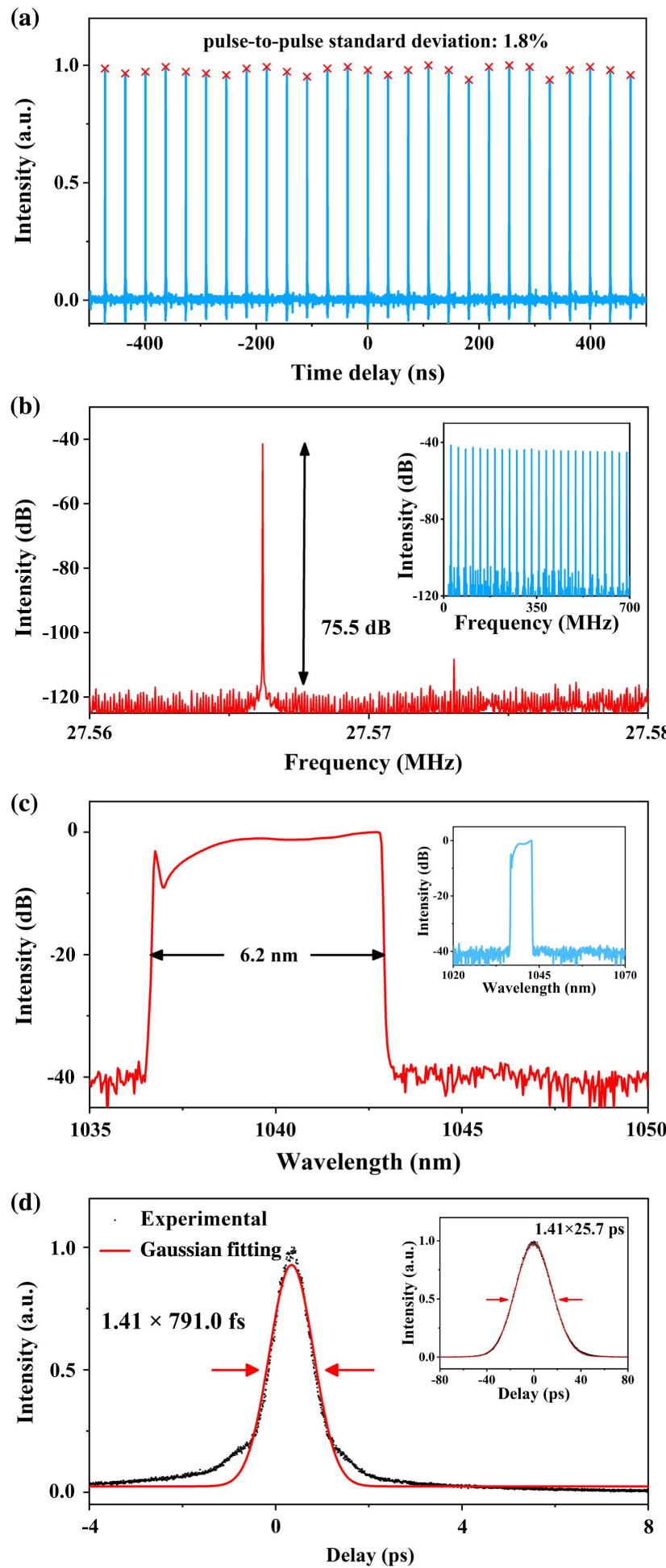

Fig. 6. Output characteristics of the laser when the pump power was $160 \mathrm{~mW}$. (a) The single-pulse mode-locked pulse train. (b) The output RF spectrum with $1 \mathrm{~Hz}$ resolution bandwidth. Inset: the output RF spectrum with $100 \mathrm{~Hz}$ resolution bandwidth. (c) The output optical spectrum with a spectral resolution of $0.04 \mathrm{~nm}$. Inset: the output optical spectrum with a spectral resolution of $0.1 \mathrm{~nm}$. (d) The autocorrelation trace of dechirped output pulses with Gaussian fitting. Inset: the autocorrelation trace of output pulses with Gaussian fitting.

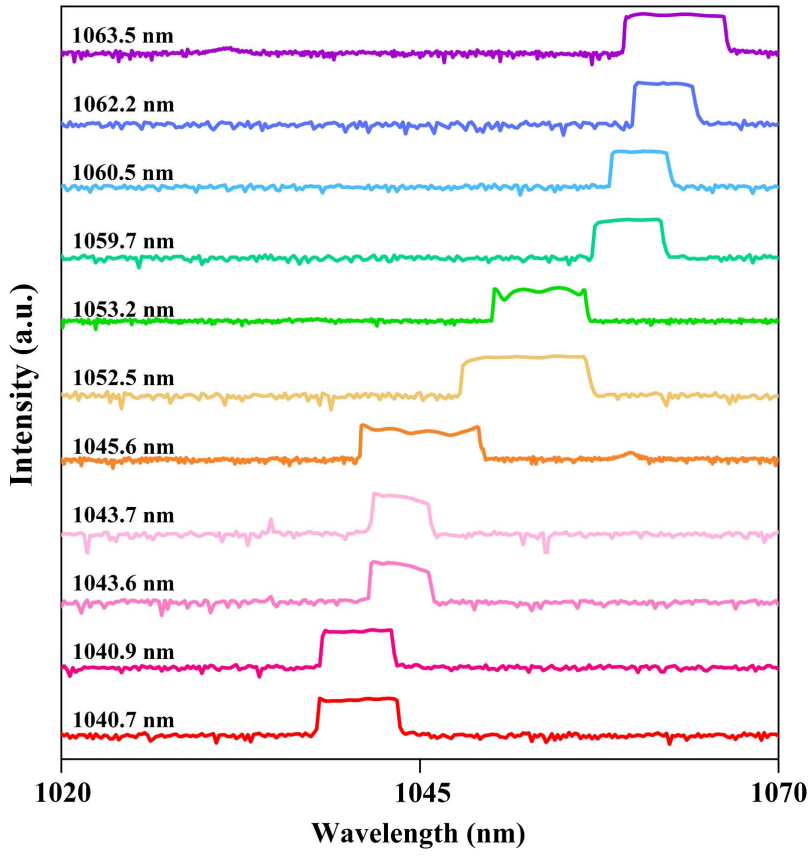

Fig. 7. Output spectra of the tunable mode-locked fiber laser with central wavelength ranging from $1040.7 \mathrm{~nm}$ to $1063.5 \mathrm{~nm}$.

is in good agreement with the simulated results shown in Fig. 2. The spectral tuning range of the mode-locking operation may be limited by the gain bandwidth of the Yb-doped fiber or bandpass filter effect induced by other fiber devices. The phenomena of tunable laser output can be inferred by the simulation results, which verified the validity of our numerical model in Section 2.

\section{Conclusion}

We have demonstrated a novel all-fiber tunable mode-locked laser with an SA based on the NL-KBC effect. The mechanisms of tunable band-pass filter and saturable absorption effects in the SA have been demonstrated by an original numerical model. Guided by the simulation results with adjusting the bending condition of the GIMF, the laser could produce stable dissipative soliton pulses with tunable central wavelengths ranging from $1033 \mathrm{~nm}$ to $1063 \mathrm{~nm}$. This work provides a new approach for constructing wavelength-tunable mode-locked fiber lasers. Future works will focus on the all-polarization-maintainingfiber configuration and precise tuning of the mode-locked wavelength in these lasers.

\section{Acknowledgement}

The authors acknowledge Zhanwei Liu for the helpful discussion on the numerical model and the MPA program. We acknowledge Ugur Tegin for helpful discussions on the laser design and Yu Duan of Emgo-tech Ltd. (Zhuhai), Fujuan Wang, and Jiaoyang Li for the technical support in this work. 


\section{References}

1. X. He, Z. Liu, and D. N. Wang, "Wavelength-tunable, passively mode-locked fiber laser based on graphene and chirped fiber Bragg grating," Opt. Lett. 37, 2394 (2012).

2. J. Schröder, T. D. Vo, and B. J. Eggleton, "Repetition-rate-selective, wavelength-tunable mode-locked laser at up to $640 \mathrm{GHz}$," Opt. Lett. 34, 3902 (2009).

3. H. Li, F. Hu, Y. Tian, P. Wang, J. Zhang, and S. Xu, "Continuously wavelength-tunable mode-locked Tm fiber laser using stretched SMF-GIMFSMF structure as both saturable absorber and filter," Opt. Express 27, 14437 (2019).

4. H. Zhang, L. Jin, Y. Xu, H. Zhang, L. Shi, T. Wang, W. Pan, and X. Ma, "Cband wavelength tunable mode-locking fiber laser based on CD-SMS structure," Appl. Opt. 58, 5788 (2019).

5. K. Krupa, A. Tonello, B. M. Shalaby, M. Fabert, A. Barthélémy, G. Millot, S. Wabnitz, and V. Couderc, "Spatial beam self-cleaning in multimode fibres," Nat. Photonics 11, 237 (2017).
6. Z. Liu, L. G. Wright, D. N. Christodoulides, and F. W. Wise, "Kerr self-cleaning of femtosecond-pulsed beams in graded-index multimode fiber," Opt. Lett. 41, 3675 (2016).

7. B. Zhang, S. Ma, Q. He, J. Guo, Z. Jiao, and B. Wang, "Investigation on saturable absorbers based on nonlinear Kerr beam cleanup effect," Opt. Express 28, 6367 (2020).

8. B. Zhang, S. Ma, S. Lu, Q. He, J. Guo, Z. Jiao, and B. Wang, "All-fiber modelocked ytterbium-doped fiber laser with a saturable absorber based on the nonlinear Kerr beam cleanup effect," Opt. Lett. 45, 6050 (2020).

9. H.F. Taylor, "Bending effects in optical fibers," J. Light. Technol. 2, 617 (1984).

10. Y. H. Sohn, "Light propagation in multimode graded-index optical fibers undergoing bending," Ph.D. dissertation (Texas A\&M University, 2002).

11. L. G. Wright, Z. M. Ziegler, P. M. Lushnikov, Z. M. Zhu, M. A. Eftekhar, D. N. Christodoulides, and F. W. Wise, "Multimode nonlinear fiber optics: massively parallel numerical solver, tutorial and outlook," IEEE J. Sel. Top. Quantum Electron. 24, 5100516 (2018).

12. https://wise.research.engineering.cornell.edu/ or https://github.com/ wiselabaep/gmmnlse-solver-final. 